\title{
OSCILLATION THEOREMS FOR LINEAR SECOND ORDER ORDINARY DIFFERENTIAL EQUATIONS ${ }^{1}$
}

\author{
J. W. MACKI AND J. S. W. WONG
}

We are here concerned with the oscillatory behavior of solutions of the equation

$$
x^{\prime \prime}+a(t) x=0,
$$

where $a(t)$ is locally integrable on $[0, \infty)$. Our main result is an extension of a nonoscillation theorem due to Hartman [2, Theorem II], the contrapositive of which is a useful criterion for equation (1) to be oscillatory. The approach here differs from that of Hartman in the use of an averaging technique recently introduced by Coles [1]. In fact, we introduce a somewhat more general notion of averaging, in terms of which we prove generalizations of results of Coles, and Howard [3].

A pair of functions $(\sigma, \alpha)$ is called an averaging pair if and only if

(i) $\sigma \in C[0, \infty), \sigma \geqq 0$ and $\alpha>0, \alpha$ is absolutely continuous on compact subintervals of $[0, \infty)$, and

$$
\lim _{T \rightarrow \infty} \int_{1}^{T} \sigma(s)\left(\int_{0}^{s} \alpha(u) \sigma^{2}(u) d u\right)^{-1} d s=+\infty .
$$

Note that (i) and (ii) imply that $\int_{0}^{\infty} \sigma(s) d s=+\infty$. Using this fact, it is easy to show that

$$
f \in L_{1}(0, \infty) \Rightarrow \lim _{T \rightarrow \infty} \sum_{T}(F)=0
$$

where

$$
F(t)=\int_{t}^{\infty} f(u) d u, \quad \sum_{T}(g)=\left(\int_{0}^{T} \sigma(s) d s\right)^{-1} \int_{0}^{T} \sigma(s) g(s) d s .
$$

Simple examples of averaging pairs are $(1,1),\left((t+1)^{-1}, t+1\right)$, $\left((t+1)^{-1 / 2}, \log (t+1)\right)$. In fact, any pair $(\sigma, \alpha)$, with $(\sigma, \alpha)$ satisfying (i), $\sigma \cdot \alpha$ bounded, and $\int_{0}^{\infty} \sigma(s) d s=\infty$, is an averaging pair.

Presented to the Society, June 17, 1967 under the title $A n$ oscillation criterion for linear second order ordinary differential equations; received by the editors June 26, 1967 and, in revised form, September 21, 1967.

1 This research was supported in part by the Defence Research Board of Canada under Grant No. 9540-11 and No. 9540-12. 
THEOREM 1. If (1) is nonoscillatory, then for any given averaging pair $(\sigma, \alpha)$ satisfying

$$
f \in L_{1}(0, \infty) \Rightarrow \lim _{T \rightarrow \infty} \sum_{T}(\alpha f)=0,
$$

we must have either

$$
\liminf _{T \rightarrow \infty} \sum_{T}\left(K_{\alpha}+\frac{\alpha^{\prime}}{2}\right)=-\infty
$$

or

$$
\lim _{T \rightarrow \infty} \sum_{T}\left(K_{\alpha}+\frac{\alpha^{\prime}}{2}\right)=C, \quad-\infty<C<\infty,
$$

and in fact, in case (b) we have the stronger conclusion

$$
\lim _{T \rightarrow \infty} \sum_{T}\left(\left(C-K_{\alpha}-\frac{\alpha^{\prime}}{2}\right)^{2}\right)=0 \text {, }
$$

where

$$
K_{\alpha}(s)=\int_{0}^{s}\left(\alpha(u) a(u)-\frac{1}{4} \frac{\left[\alpha^{\prime}(u)\right]^{2}}{\alpha(u)}\right) d u .
$$

It is easy to verify that the simple averaging pairs mentioned above all satisfy (iii). However, condition (iii) is rather intangible as it stands, and in practice we use the following condition, which, in conjunction with conditions (i) and (ii), implies (iii):

$$
\sigma(t) \alpha(t)=O\left(\int_{0}^{t} \sigma(u) d u\right), \quad \text { as } t \rightarrow \infty .
$$

To see that (iii)' implies (iii) when $(\sigma, \alpha)$ is an averaging pair, suppose

$$
\sigma(t) \alpha(t) \leqq K_{1} \int_{0}^{t} \sigma(s) d s \equiv K_{1} S(t),
$$

for $t \geqq B$ and $K_{1}$ some positive constant. Observe that

$$
\begin{aligned}
\left|\int_{0}^{T} \sigma(s) \alpha(s) f(s) d s\right| & \leqq \int_{0}^{B} \sigma(s) \alpha(s)|f(s)| d s+K_{1} \int_{B}^{T}|f(s)| S(s) d s \\
& \leqq K_{2}+K_{1} \int_{B}^{T} \sigma(s) \int_{s}^{\infty}|f(u)| d u d s
\end{aligned}
$$

where $K_{2}$ is some appropriate constant. Using (2) and (3), one easily obtains (iii). 
If for some averaging pair, $(\sigma, \alpha)$, satisfying (iii), we have

$$
\liminf _{T \rightarrow \infty} \sum_{T}\left(K_{\alpha}+\frac{\alpha^{\prime}}{2}\right)>-\infty,
$$

and

$$
\limsup _{T \rightarrow \infty} \sum_{T}\left(K_{\alpha}+\frac{\alpha^{\prime}}{2}\right)=+\infty,
$$

then Theorem 1 shows that (1) is oscillatory. In the particular case $\lim \sup =\lim \inf =+\infty$, as considered in [1] and [4], $(\sigma, \alpha)$ need not satisfy (iii), and we have the following result:

THEOREM 2. If there exists an averaging pair, $(\sigma, \alpha)$, such that

$$
\lim _{T \rightarrow \infty} \sum_{T}\left(K_{\alpha}+\frac{\alpha^{\prime}}{2}\right)=+\infty,
$$

then equation (1) is oscillatory.

If we take $\alpha \equiv \sigma \equiv 1$ in Theorem 2, we obtain the well-known theorem of Wintner [4], and if we consider only averaging pairs of the form $(\sigma, 1)$, we obtain Coles' Theorem. If we restrict ourselves to averaging pairs of the form $(1 / \alpha, \alpha)$ with $\int^{\infty}(1 / \alpha(s)) d s=+\infty$, we obtain extensions of results due to Howard. (It should be mentioned, however, that Howard was primarily interested in nonlinear equations.) Howard's assertion that his results (when specialized to linear equations) cover the results of Opial [5] appears to be in error, since even our stronger results do not (as far as we know) cover those of Opial.

If we restrict ourselves to the single averaging pair $(1,1)$ in Theorem 1, we obtain Hartman's Theorem. Although the Wintner Theorem and its extensions (e.g., the Coles and Opial results) are easier to apply in practice, the Hartman Theorem appears to us to be a considerably deeper result. For example, neither Wintner's nor Coles' Theorem yields any information when

$$
\limsup _{T \rightarrow \infty} \int_{0}^{T} a(u) d u<\infty .
$$

On the other hand, when $a(t)=\sin t$, Hartman's Theorem shows that (1) is oscillatory. In fact, Opial's Theorem (if $\lim _{t \rightarrow \infty} K_{\omega}(t)=+\infty$ for some $\omega \geqq 0, \omega \in C^{\prime}[0, \infty)$, then (1) is oscillatory) does not cover this case, either.

We now give a simple example of an equation for which none of the results quoted above (including the Hartman Theorem) gives any 
information, while Theorem 2 shows that it is oscillatory. Consider

$$
x^{\prime \prime}+\frac{\cos t}{(\log (t+1))^{1 / 2}} x=0 .
$$

Since there are arbitrarily large $t$ for which $a(t)=0$, Opial's condition does not hold, and since the improper integral $\int_{0}^{\infty} a(u) d u$ converges in this case, it is clear that neither Coles' nor Hartman's result applies. We shall determine an averaging pair $(\sigma, \alpha)$ such that (4) holds -hence, by Theorem 2, (5) is oscillatory. We choose $\alpha(t)=\log (t+1)$, and then compute

$$
\begin{aligned}
K_{\alpha}(t) & =\int_{0}^{t}(\log (u+1))^{1 / 2} \cos u d u+O(1) \\
& =(\log (t+1))^{1 / 2} \sin t+O(1) .
\end{aligned}
$$

We now define $\sigma(t)=(t+1)^{-(1 / 2)} \chi(t)$, where $\chi(t)$ is the characteristic function of $\left\{t: K_{\alpha}(t) \geqq 0\right\}$. From (6), it is clear that $\chi(t)$ "looks like" the characteristic function of the set $\{t: \sin t \geqq 0\}$, i.e.

$$
\begin{aligned}
\chi(t) & =0, & & (2 k-1) \pi+\delta(k) \leqq t \leqq 2 k \pi-\delta(k), \\
& =1, & & 2 k \pi+\delta(k) \leqq t \leqq(2 k+1) \pi-\delta(k),
\end{aligned}
$$

$k=1,2,3, \cdots$ where $\lim _{k \rightarrow \infty} \delta(k)=0$. Using this fact, it is easy to show that if $f$ is any positive monotone decreasing function on $[0, \infty)$, then

$$
\int_{0}^{\infty} f(t) d t=+\infty \Rightarrow \int_{0}^{\infty} f(t) \chi(t) d t=+\infty,
$$

from which (ii) and (4) follow by routine computations.

Proof OF Theorem 1. Let $x(t)$ be any solution of (1) with $x(t) \neq 0$ for $t \geqq t_{0}$. For any given averaging pair $(\sigma, \alpha)$ set $z=\alpha x^{\prime} / x$ for $t \geqq t_{0}$. Suppose that (a) does not hold. We shall show that (b) must hold. We first have

$$
z^{\prime}(t)+\frac{1}{\alpha(t)}\left(z(t)-\frac{\alpha^{\prime}(t)}{2}\right)^{2}+\left(\alpha(t) a(t)-\frac{\left(\alpha^{\prime}(t)\right)^{2}}{4 \alpha(t)}\right)=0 .
$$

Define $v(t)=z(t)-\alpha^{\prime}(t) / 2$, and integrate (7) to get

$$
v(t)+\int_{t_{0}}^{t} \frac{v^{2}(s)}{\alpha(s)} d s+K_{\alpha}(t)+\frac{\alpha^{\prime}(t)}{2}=C_{0},
$$

where $C_{0}=z\left(t_{0}\right)+K_{\alpha}\left(t_{0}\right)$. From (8), we observe that when (a) does not hold, 


$$
\sum_{T}(v)+\sum_{T}\left(\int_{t_{0}}^{t} \frac{v^{2}}{\alpha}\right)=C_{0}-\sum_{T}\left(K_{\alpha}+\frac{\alpha^{\prime}}{2}\right)<\infty .
$$

We claim that this implies $\int_{t_{0}}^{\infty}\left(v^{2}(s) / \alpha(s)\right) d s<\infty$. Suppose not, then it is easy to see that

$$
\lim _{T \rightarrow \infty} \sum_{T}\left(\int_{t_{0}}^{t}\left(v^{2} / \alpha\right)\right)=+\infty .
$$

From (9), it follows that there exists $T_{0} \geqq t_{0}$ such that

$$
\left|\int_{t_{0}}^{t} \sigma(s) v(s) d s\right|>\frac{1}{2} \int_{t_{0}}^{t} \sigma(s) \int_{t_{0}}^{s} \frac{v^{2}(u)}{\alpha(u)} d u, \quad t \geqq T_{0} .
$$

If we use the Cauchy-Schwarz inequality to estimate the left-hand side of (10), and define

$$
W(t)=\int_{t_{0}}^{t} \sigma(s) \int_{t_{0}}^{s} \frac{v^{2}(u)}{\alpha(u)} d u d s,
$$

then we obtain the following differential inequality for $W$ :

$$
4 \frac{W^{\prime}(t)}{W^{2}(t)} \geqq \sigma(t)\left(\int_{t_{0}}^{t} \alpha(s) \sigma^{2}(s) d s\right)^{-1}, \quad t \geqq T_{0} .
$$

An integration of (11) yields

$$
\frac{4}{W\left(T_{0}\right)}-\frac{4}{W(T)} \geqq \int_{T_{0}}^{T} \sigma(t)\left(\int_{t_{0}}^{t} \alpha(s) \sigma^{2}(s) d s\right)^{-1} d t
$$

which with condition (ii), produces the desired contradiction.

Since $\int_{t_{0}}^{\infty}\left(v^{2}(s) / \alpha(s)\right) d s=C_{1}<\infty$, we can rewrite (8) in the following form:

$$
v(t)-\int_{t}^{\infty} \frac{v^{2}(s)}{\alpha(s)} d s=C_{0}-C_{1}-K_{\alpha}(t)-\frac{\alpha^{\prime}(t)}{2} .
$$

Squaring (12), multiplying by $\sigma(t)$, and integrating, we obtain

$$
\sum_{T}\left((v-V)^{2}\right)=\sum_{T}\left(\left(C-K_{\alpha}-\frac{\alpha^{\prime}}{2}\right)^{2}\right),
$$

where $V(t)=\int_{2}^{\infty}\left(v^{2}(s) / \alpha(s)\right) d s$ and $C=C_{0}-C_{1}$. Note that

$$
\sum_{T}\left((v-V)^{2}\right) \leqq 2 \sum_{T}\left(v^{2}\right)+2 \sum_{T}\left(V^{2}\right)
$$


From (2), we have $\lim _{T \rightarrow \infty} \sum_{T}\left(V^{2}\right)=0$, and since the averaging pair $(\sigma, \alpha)$ satisfies property (iii), we have

$$
\lim _{T \rightarrow \infty} \sum_{T}\left(v^{2}\right)=\lim _{T \rightarrow \infty} \sum_{T}\left(\alpha \cdot \frac{v^{2}}{\alpha}\right)=0 .
$$

The conclusion that (b) holds follows from (13) and (14).

Proof of Theorem 2. The proof follows that of Theorem 1 up to and including the fact that $\int^{\infty} v^{2} / \alpha<\infty$. (Note that property (iii) is not used in this portion of the proof of Theorem 1.) Returning to equation (9) and using (4), we obtain again (10) and (11). Repeating the argument as before by integrating (11), we obtain the desired contradiction. Hence, all solutions of (1) are oscillatory.

\section{REFERENCES}

1. W. J. Coles, An oscillation criterion for second-order linear differential equations, Proc. Amer. Math Soc. 19 (1968), 755-759.

2. P. Hartman, On non-oscillatory linear differential equations of second order, Amer. J. Math. 74 (1952), 389-400.

3. H. C. Howard, Oscillation and nonoscillation criteria for $y^{\prime \prime}+f(y(x)) p(x)=0, \mathrm{~Pa}$ cific J. Math. 12 (1962), 243-251.

4. A. Wintner, A criterion of oscillatory stability, Quart. Appl. Math. 7 (1949) $115-117$.

5. Z. Opial, Sur un critere d'oscillation des integrales de l'equation differentielle $\left(Q(t) x^{\prime}\right)^{\prime}+f(t) x=0$, Ann. Polon. Math. 6 (1959), 99-104.

University of Alberta and

Mathematics Research Center, University of Wisconsin 